\title{
Measurement and tricubic interpolation of the magnetic field for the OLYMPUS experiment
}

\author{
J. C. Bernauer ${ }^{\mathrm{a}}$, J. Diefenbach ${ }^{\mathrm{b}, 1}$, G. Elbakianc ${ }^{\mathrm{c}}$, G. Gavrilov ${ }^{\mathrm{d}}$, N. Goerrissen ${ }^{\mathrm{e}}$, D. K. Hasell ${ }^{\mathrm{a}}$, B. S. Henderson ${ }^{\mathrm{a}, *}$, Y. Holler ${ }^{\mathrm{e}}$,
}

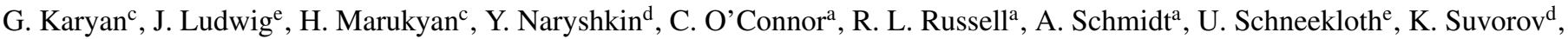
D. Veretennikov ${ }^{\mathrm{d}}$

\author{
${ }^{a}$ Massachusetts Institute of Technology, Laboratory for Nuclear Science, Cambridge, MA, USA \\ ${ }^{b}$ Hampton University, Hampton, VA, USA \\ ${ }^{c}$ Alikhanyan National Science Laboratory (Yerevan Physics Institute), Yerevan, Armenia \\ ${ }^{d}$ Petersburg Nuclear Physics Institute, Gatchina, Russia \\ ${ }^{e}$ Deutsches Elektronen-Synchrotron DESY, Hamburg, Germany
}

\begin{abstract}
The OLYMPUS experiment used a $0.3 \mathrm{~T}$ toroidal magnetic spectrometer to measure the momenta of outgoing charged particles. In order to accurately determine particle trajectories, knowledge of the magnetic field was needed throughout the spectrometer volume. For that purpose, the magnetic field was measured at over 36,000 positions using a three-dimensional Hall probe actuated by a system of translation tables. We used these field data to fit a numerical magnetic field model, which could be employed to calculate the magnetic field at any point in the spectrometer volume. Calculations with this model were computationally intensive; for analysis applications where speed was crucial, we pre-computed the magnetic field and its derivatives on an evenly spaced grid so that the field could be interpolated between grid points. We developed a spline-based interpolation scheme suitable for SIMD implementations, with a memory layout chosen to minimize space and optimize the cache behavior to quickly calculate field values. This scheme requires only one-eighth of the memory needed to store necessary coefficients compared with a previous scheme [1]. This method was accurate for the vast majority of the spectrometer volume, though special fits and representations were needed to improve the accuracy close to the magnet coils and along the toroid axis.
\end{abstract}

Keywords:

OLYMPUS, magnet, Hall probe, survey, 3D interpolation, tricubic spline

\section{Introduction}

OLYMPUS is a particle physics experiment comparing the elastic cross section for positron-proton scattering to that of electron-proton scattering [2]. This measurement has been of interest recently because it tests the hypothesis that hard twophoton exchange is responsible for the proton form factor discrepancy [3, 4]. OLYMPUS took data in 2012 and 2013 at the DORIS storage ring at DESY, in Hamburg, Germany. During data taking, beams of electrons and positrons were directed through a windowless hydrogen gas target. The scattered lepton and recoiling proton from elastic scattering events were detected in coincidence with a toroidal magnetic spectrometer. The spectrometer's support structure, magnet, and several subdetectors were originally part of the BLAST experiment [5]. Several new detectors were specially built for OLYMPUS to serve as luminosity monitors. A schematic of the apparatus is shown in Figure 1.

The OLYMPUS spectrometer used a magnetic field for two purposes. First, the field produced curvature in the trajectories

\footnotetext{
${ }^{*}$ Corresponding author

Email address: bhender1@mit.edu (B. S. Henderson)

${ }^{1}$ Currently with Johannes Gutenberg-Universität, Mainz, Germany
}

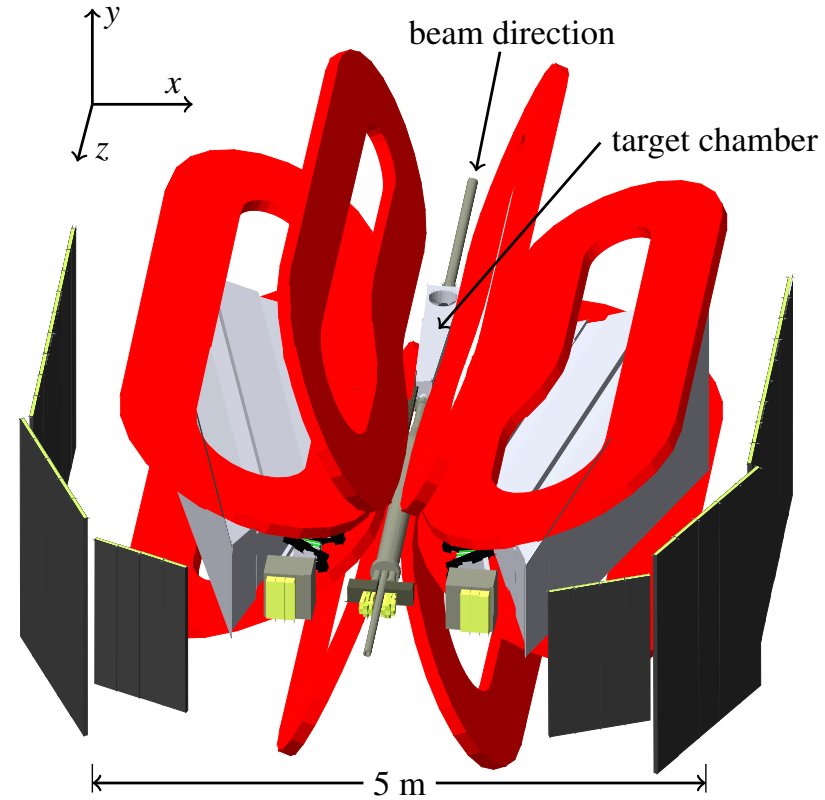

Figure 1: This schematic shows how the eight magnet coils are situated around the OLYMPUS detectors. 
of charged particles so that the detectors could measure their ${ }_{59}$ momentum. Typical momenta of particles from elastic scattering reactions ranged from 0.2 to $2 \mathrm{GeV} / c$, corresponding to ${ }^{60}$ sagittas as small as $5 \mathrm{~mm}$ in the OLYMPUS tracking detectors. ${ }^{61}$ Secondly, the magnet acted as a filter, preventing low-energy ${ }^{62}$ charged particles (from background processes like Møller or ${ }^{63}$ Bhabha scattering) from reaching the tracking detectors.

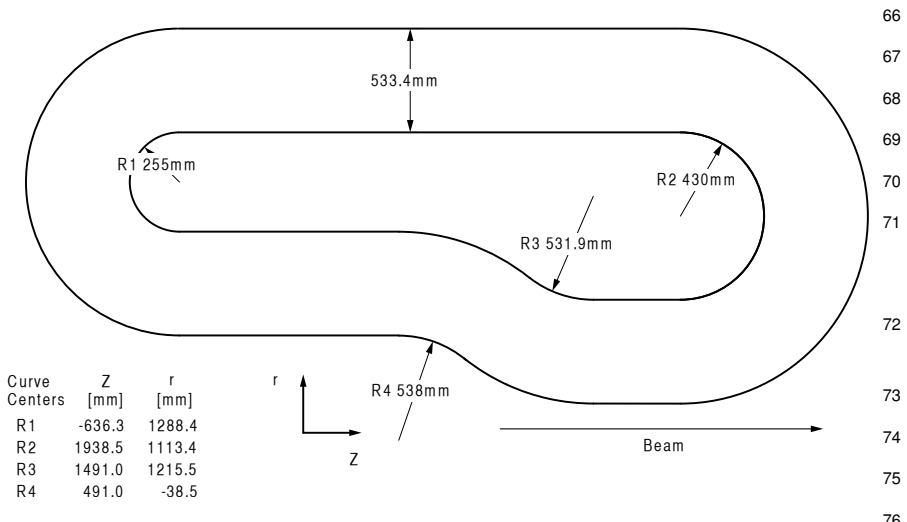

Figure 2: Individual magnet coils were narrower at the upstream end to accom- 77 modate the target chamber. shape, each with 26 windings of hollow copper bars potted to- ${ }^{81}$ gether with epoxy. The coil shape is shown in Figure 2. The coils were arranged to produce a toroidal field, with the beamline passing down the symmetry axis of the toroid. During OLYMPUS running, the coils carried $5000 \mathrm{~A}$ of current, which produced a peak field of approximately $0.3 \mathrm{~T}$.

Knowledge of the spectrometer's magnetic field was necessary for reconstructing particle trajectories through the OLYMPUS spectrometer. However, calculating the field using the design drawings and the Biot-Savart law was not feasible for two reasons. First, the positions of the copper bars within the epoxy were not well known. Secondly, the coils were observed to deform slightly when current passed through them due to magnetic forces. Instead, at the conclusion of data taking, extensive field measurements were made of the magnet in situ. A measurement apparatus, consisting of a three-dimensional Hall probe actuated by a system of translation tables, was used to measure the magnetic field vector at over 36,000 positions in both the left- and right-sector tracking volumes.

This paper presents both the measurement technique and the 84 subsequent data analysis used to characterize the field of the 85 OLYMPUS magnet. Section 3 describes the measurement ap- 86 paratus, the measurement procedure, and the techniques used 87 to establish the Hall probe position. Section 4 describes how 88 we fit the magnetic field data with a numerical field model to 89 allow us to calculate the field at positions we did not measure. 90 Section 5 describes the special interpolation scheme we devel- 91 oped to facilitate rapid queries of the magnetic field. Finally, 92 Section 6 describes the special modifications to our field model ${ }_{93}$ that were needed in two regions where the model did not per- 94 form adequately.
The OLYMPUS magnet consisted of eight coils, identical in ${ }^{80}$

\section{Coordinate System}

This paper makes frequent references to positions and directions in the standard OLYMPUS coordinate system. In this system, the $x$-axis points left from the beam direction, the $y$-axis points up, and the $z$-axis points in the direction of the beam. The coordinate origin is taken to be the center of the target. OLYMPUS has two sectors of detectors, which lie to the left $(x>0)$ and right $(x<0)$ of the beamline, centered on the $y=0$ plane. Since the magnet has toroidal symmetry, it is sometimes convenient to work with cylindrical coordinates. We use $r$ to refer to the radius from the $z$ axis and $\phi$ to refer to the azimuthal angle from the $x z$ plane. For example, a point on the positive $y$ axis lies at $\phi=90^{\circ}$.

\section{Field Measurements}

The magnetic field measurements at OLYMPUS were more involved than those made during the BLAST experiment, detailed in a previous article [6]. Like at BLAST, preliminary field measurements along the beamline were made to align the coils during the toroid's assembly; in addition, a detailed survey was made after data taking was complete. This was important because OLYMPUS compared scattering with electrons to scattering with positrons; the magnetic field introduces differences in trajectories between the two species. Field inaccuracies directly contribute to the systematic error.

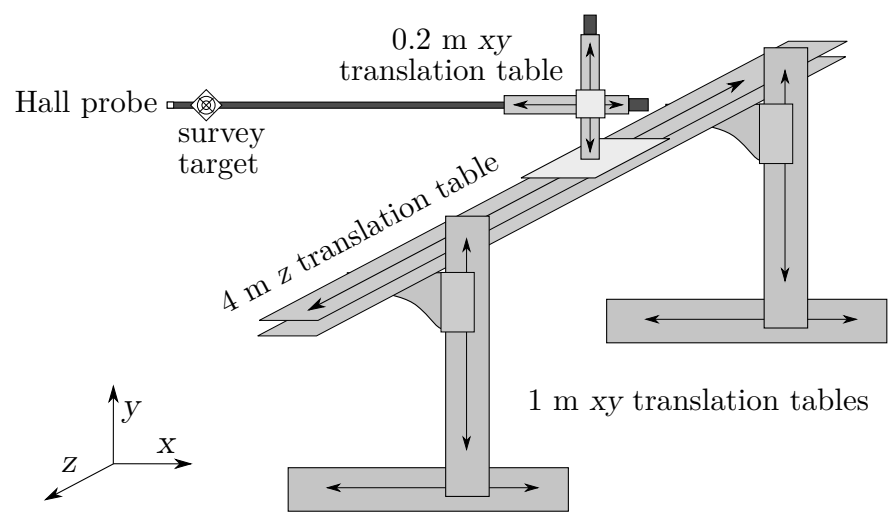

Figure 3: Two $1 \mathrm{~m} x y$ tables supported a long $x y z$ table, which could scan $4 \mathrm{~m}$ in the $z$ direction. In this configuration, the apparatus is assembled to measure the field on the $x>0$ side of the toroid.

The measurement apparatus was built from a system of translation tables, a schematic of which is shown in Figure 3. The apparatus was originally built to measure the field of the undulator magnets of the FLASH free electron laser at DESY [7]. The entire apparatus was supported by a pair of two-dimensional translation stands, which had $1 \mathrm{~m}$ of range in the $x$ and $y$ directions. These stands were moved synchronously and acted as a single table. This table supported a three-dimensional translation table with $4 \mathrm{~m}$ of range in the $z$ direction and $0.2 \mathrm{~m}$ of range in the $x$ and $y$ directions. This system of translation tables was used to move a three-dimensional Hall probe at the end of a carbon fiber rod, held parallel to the $x$ axis. The range of motion in $x$ and $y$ was extended beyond the $1.2 \mathrm{~m}$ range of the translation 
tables by using rods of different lengths and different brackets to connect the rods to the tables. To allow the Hall probe and rod to move through the magnet volume without obstructions, the detectors and parts of the beamline were removed before the apparatus was assembled.

The position of the Hall probe was monitored using a theodolite equipped with a laser range-finder. The theodolite could determine the relative position to a reflective target, which was attached to the measurement rod. The theodolite's position was calibrated daily by surveying a set of reference points on the walls of the experimental hall and on the frame of the magnet, the positions of which were established by previous surveys. ${ }^{130}$

Measurement scans were made by moving to a desired $x$ and ${ }^{131}$ $y$ position and then stepping along the $z$ direction. At the start- ${ }^{132}$ ing and ending point of a scan, the theodolite was used to sur- ${ }^{133}$ vey the position of the reflective target. At each step in the ${ }^{134}$ scan, the probe would be moved to the new $z$ position, followed ${ }^{135}$ by a pause of one second to allow any vibrations in the rod ${ }^{136}$ to dampen. Then, a measurement was made, and the probe was stepped to the next point. This procedure was computercontrolled using a LabVIEW application. Measurements were made in a three-dimensional grid with $50 \mathrm{~mm}$ spacing within $1 \mathrm{~m}$ of the beamline and $100 \mathrm{~mm}$ spacing elsewhere. Measurement scans were made along as many $x, y$ trajectories as could be reached, given the ranges of the rods and tables, while still avoiding collisions between the magnet and the measurement apparatus. The nominal probe positions for each scan are shown in Figure 4. The field was measured at over 36,000 points across both sectors.

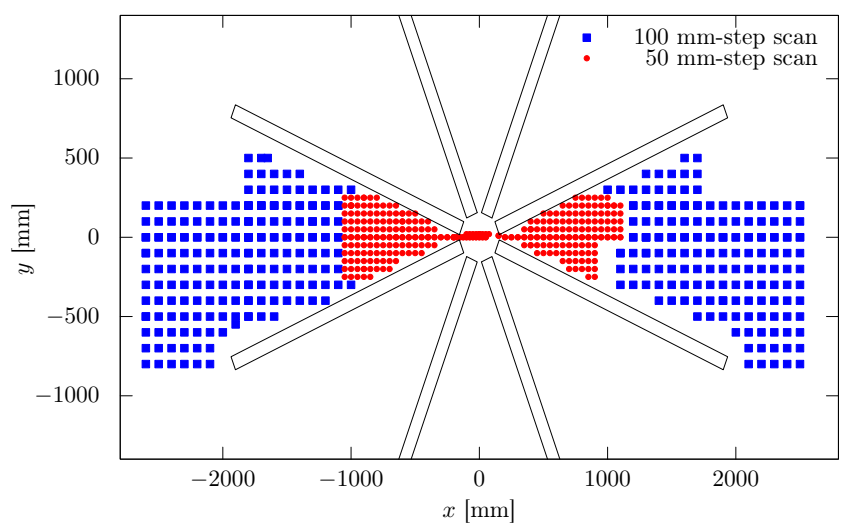

Figure 4: Magnetic field measurements were made with greater density in the inner part of the tracking volume where the field gradients are highest.

After surveying the left sector, the apparatus was taken apart and reassembled for measurements of the right sector. The field in the beamline region was measured from both the left and right. These overlapping measurements were consistent in all three field directions at a level better than $5 \times 10^{-5} \mathrm{~T}$.

Measurements with the theodolite confirmed that the translation tables did not provide the millimeter-level accuracy desired. Over the course of $4 \mathrm{~m}$ of translation in $z$, the long table introduced perturbations of several millimeters in $x$ and $y$. The position measurements from the theodolite were used to cor- rect for these perturbations and recover the true position of the Hall probe. Every time a new rod was installed on the tables, a calibration scan was made, in which the reflective target was surveyed at every step in the $4 \mathrm{~m} z$ translation. This allowed us to determine the three-dimensional trajectory of the target. We found that the trajectories had similar shapes for all rods, and the motion could be parameterized with:

$$
\begin{aligned}
& x(z)=x_{t}(z)+L_{r} \cos \left(\theta_{t}(z)\right)+\text { linear term } \\
& y(z)=y_{t}(z)+L_{r} \sin \left(\theta_{t}(z)\right)+\text { linear term }
\end{aligned}
$$

where $x_{t}, y_{t}$, and $\theta_{t}$ represent perturbation functions that are common to the table (independent of which rod was used), and $L_{r}$ is the length of the specific rod in use. A linear term was used to match the start and end points of the trajectory to the starting and ending positions of each measurement scan, as surveyed by the theodolite. Figure 5 shows data from three calibration scans fit using this parameterization.

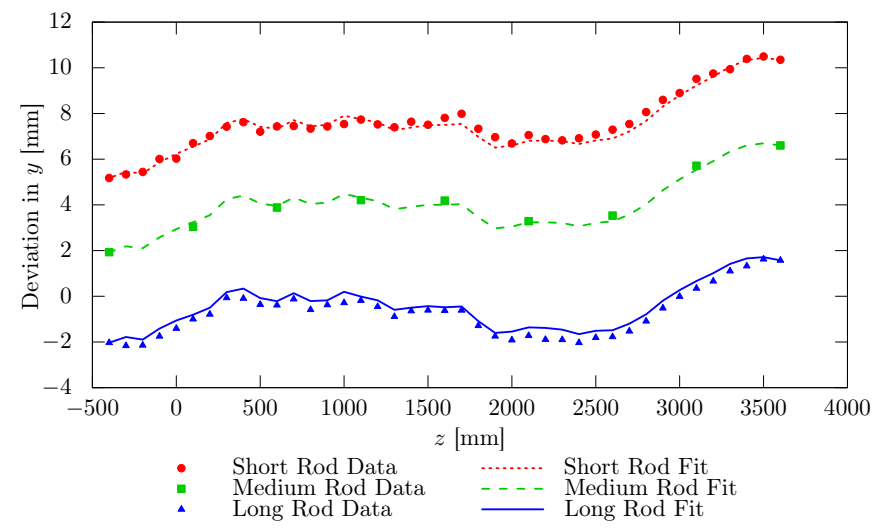

Figure 5: The translation tables deviated from their nominal positions over the length of a scan, but these deviations could be fit with a simple parameterization.

\section{Coil Fitting}

The magnetic field measurement data were fit using a numerical magnetic field model, thereby solving two problems. First, the model can be used to calculate the magnetic field in regions where measurements could not be made, i.e., where the probe was obstructed. Secondly, any measurements with errant field readings or offset position assignments have their local influence dampened in the model.

In the magnetic field model, the current in each copper winding was approximated by a sequence of line segments. The magnetic field produced by a line segment of current was found by integrating the Biot-Savart law. The magnetic field at position $\vec{x}$ produced by a line segment starting at position $\vec{p}_{1}$ and ending at $\vec{p}_{2}$, carrying current $I$, is given by:

$$
\begin{gathered}
\vec{B}=\frac{\mu_{0} I}{4 \pi} \frac{\vec{L} \cdot\left(\hat{V}_{2}-\hat{V}_{1}\right)}{L^{2}(\vec{c}-\vec{x}) \cdot(\vec{c}-\vec{x}-\vec{L} / L)} \vec{L} \times(\vec{c}-\vec{x}) \\
\vec{c}=\left(\vec{p}_{1}+\vec{p}_{2}\right) / 2
\end{gathered}
$$




$$
\begin{aligned}
\vec{L} & =\vec{p}_{2}-\vec{p}_{1} \\
\hat{V}_{i} & =\frac{\vec{p}_{i}-\vec{x}}{\left|\vec{p}_{i}-\vec{x}\right|} .
\end{aligned}
$$

The OLYMPUS coils have straight sections, easily described by line segments, and curved sections, in which the curves are approximately circular arcs. We approximated the arcs in the model by connecting multiple line segments to form a polygon. To divide an arc subtending angle $\alpha$ into $N$ segments, one can place $(N+1)$ vertices evenly along the arc, and connect them to form a polygon. However, we found we could better match the magnetic field of the arc by placing the vertices slightly outside of the arc, so that the polygon and arc had equal area, and thus equal dipole moments. In our approximation, we chose to start the first segment at the beginning of the arc, and to end the last segment at the end of the arc to maintain continuity. We displaced the $(N-1)$ intermediate vertices radially outward from the arc radius $R$ to $R^{\prime}$, given by:

$$
R^{\prime}=\frac{R}{N-2}\left[\sqrt{1+\frac{\alpha(N-2)}{\sin \left(\frac{\alpha}{N}\right)}}-1\right] .
$$

To fit the magnetic field model to the measurements, several ${ }^{182}$ parameters were allowed to vary, and a best fit was found by ${ }^{183}$ minimizing the sum of the squared residuals $\sum\left|\vec{B}_{\text {meas. }}-\vec{B}_{\text {model }}\right|^{2} .^{184}$ Several attempts were made in order to strike a balance between ${ }^{185}$ giving the model sufficient freedom to explain the data and in- ${ }_{186}$ troducing degrees of freedom that were unconstrained by the measurements. Ultimately, a model with 35 free parameters ${ }^{187}$ was chosen. The four coils that immediately surrounded the measurement region were each given six degrees of freedom ${ }_{189}$ (three for positioning and three for orientation). The remaining four coils were positioned around a common toroid axis (three parameters to specify the origin and three parameters to specify the orientation), but were allowed to vary collectively in radius and in-plane rotation angle. All of the coils were collectively given two degrees of freedom to stretch or compress in both in-plane directions, since the coils were observed to deform slightly when current was passed through them. The final free parameter was the current carried in the magnet, which reproduced the measured input current to within $0.1 \%$. In the final fit, arcs were divided into one line segment per degree of curvature.

The model with best-fit parameters had an r.m.s. residual: ${ }^{19}$ $\sqrt{\frac{1}{3 N} \sum\left|\vec{B}_{\text {meas. }}-\vec{B}_{\text {model }}\right|^{2}}=1.873 \times 10^{-3} \mathrm{~T}$, where $N$ describes the total number of measurement points. The residuals were not uniformly distributed over the measurement region and do not represent Gaussian errors. Many systematic effects contribute to the residuals, including any errors in determining the true probe position and inadequacy of the magnetic field model. The magnetic field generated by the model with the best fit parameters is shown in Figure 6.

\section{Interpolation}

The model calculation described in the previous section was too slow to be used directly for simulating or reconstructing

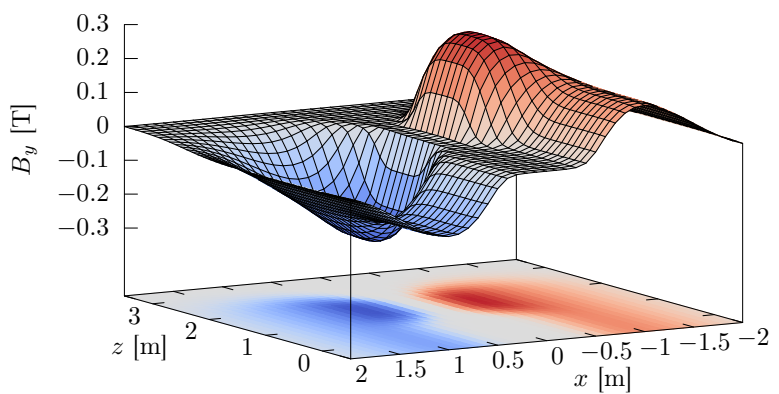

Figure 6: The magnetic field in the $y$ direction is shown for the $y=0$ plane.

particle trajectories for the OLYMPUS analysis, and so a fast interpolation scheme was developed. The coil model was used to pre-calculate the magnetic field vector and its spatial derivatives on a regular $50 \mathrm{~mm} \times 50 \mathrm{~mm} \times 50 \mathrm{~mm}$ grid covering the entire spectrometer volume, so that the field could be interpolated between surrounding grid points.

The interpolation scheme had to balance several competing goals:

- Minimizing the memory needed to store the field grid

- Minimizing computation time for field queries

- Faithfully reproducing the coil model in both the field and its derivatives.

To achieve this, an optimized tricubic spline interpolation scheme was developed based on the routine of Lekien and Marsden [1]. For each point $P$ in the grid, 24 coefficients were calculated using the coil model ( 8 per component of the vector magnetic field):

$$
\begin{array}{r}
C_{i, P}=\left\{B_{i}, \frac{\partial B_{i}}{\partial x}, \frac{\partial B_{i}}{\partial y}, \frac{\partial B_{i}}{\partial x \partial y}, \frac{\partial B_{i}}{\partial z}, \frac{\partial B_{i}}{\partial x \partial z}, \frac{\partial B_{i}}{\partial y \partial z}, \frac{\partial B_{i}}{\partial x \partial y \partial z}\right\} \\
\text { for } i \in\{x, y, z\}
\end{array}
$$

using numerical differentiation to compute the required derivatives from the coil model.

For the interpolation, it is convenient to consider the grid in terms of boxes defined by eight grid points, as shown in Figure 7 , and define box-fractional coordinates $x_{f}, y_{f}, z_{f} \in[0,1]$ parallel to the global axes spanning each box. Each point in the grid is labeled with an integer index $j$, such that stepping from point $P_{j}$ one unit in $x$ reaches point $P_{j+1}$. Stepping one unit in $y$ from point $P_{j}$ reaches $P_{j+n_{x}}$, where $n_{x}$ is the size of the grid in the $x$ direction. Stepping from point $P_{j}$ one unit in $z$ reaches point $P_{j+n_{x} n_{y}}$, where $n_{y}$ is the size of the grid in $y$ direction. Then, a local tricubic spline can be defined for each field component in the box:

$$
B_{i}(x, y, z)=\sum_{l, m, n=0}^{3} a_{i, l m n} x_{f}^{l} y_{f}^{m} z_{f}^{n} \quad i \in\{x, y, z\},
$$


where the coefficients $\left\{a_{i, l m n}\right\}$ are functions of the set of the 64 parameters $\left\{C_{i, P}\right\}$, where $P$ is any of the eight grid points at the vertices of the box. This function is a 64-term polynomial for each box and is $C^{1}$ at the box boundaries. The coefficients $\{a\}$ can be computed from the parameters $C_{i, P}$ following the prescription in Lekien and Marsden. However, this prescription requires three $64 \times 64$ matrix multiplications per box. Once completed for a given grid box, these multiplications can be stored for future use, but this adds to the size of the grid in memory, approaching a factor of 8 for large grids.

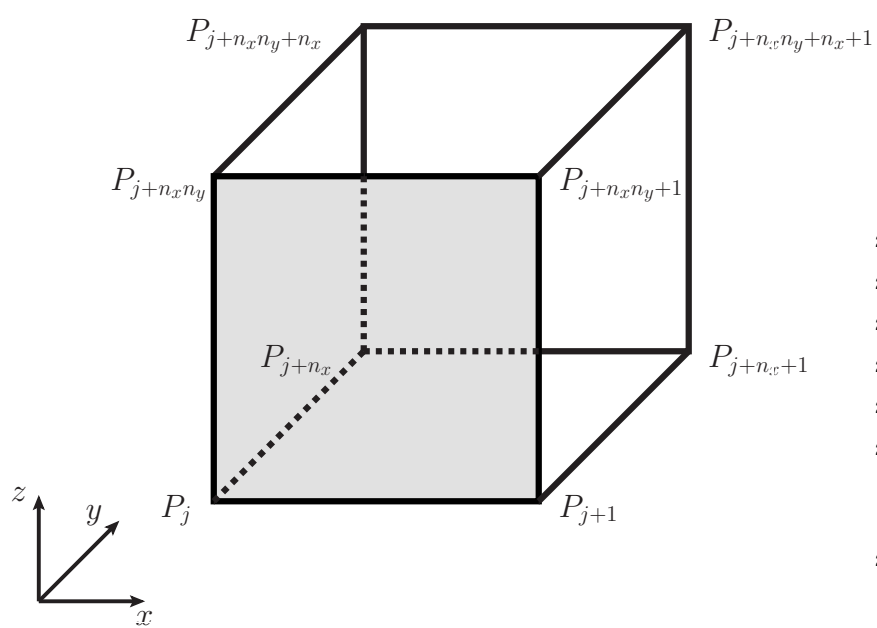

Figure 7: A generalized box in the interpolation grid identified by its lowest-222 indexed grid point $P_{j}$, where $n_{x}$ and $n_{y}$ are the $x$ and $y$ dimensions of the $\operatorname{grid}_{223}$ in units of grid points.

To avoid these costs, the spline was refactored so that the $\mathrm{pa}^{-224}$ rameters $C_{i, P}$ can be used directly as coefficients. We introduce225 the following basis functions:

$$
\begin{gathered}
f_{0}\left(x_{i}\right)=\left(x_{i}-1\right)^{2}\left(2 x_{i}+1\right) \\
f_{1}\left(x_{i}\right)=x_{i}\left(x_{i}-1\right)^{2} \\
f_{2}\left(x_{i}\right)=x_{i}^{2}\left(3-2 x_{i}\right) \\
f_{3}\left(x_{i}\right)=x_{i}\left(x_{i}-1\right)
\end{gathered}
$$

where $i \in\left\{x_{f}, y_{f}, z_{f}\right\}$. The interpolation then takes the form:

$$
B_{i}(x, y, z)=\sum_{l, m, n=0}^{3} b_{i, l m n} f_{l}\left(x_{f}\right) f_{m}\left(y_{f}\right) f_{n}\left(z_{f}\right) \quad i \in\{x, y, z\}
$$

\begin{tabular}{l|cccccccc} 
& $B_{i}$ & $\frac{\partial B_{i}}{\partial x}$ & $\frac{\partial B_{i}}{\partial y}$ & $\frac{\partial B_{i}}{\partial x \partial y}$ & $\frac{\partial B_{i}}{\partial z}$ & $\frac{\partial B_{i}}{\partial x \partial z}$ & $\frac{\partial B_{i}}{\partial y \partial z}$ & $\frac{\partial B_{i}}{\partial x \partial y \partial z}$ \\
\hline$P_{j}$ & 000 & 100 & 010 & 110 & 001 & 101 & 011 & 111 \\
$P_{j+1}$ & 200 & 300 & 210 & 310 & 201 & 301 & 211 & 311 \\
$P_{j+n_{x}}$ & 020 & 120 & 030 & 130 & 021 & 121 & 031 & 131 \\
$P_{j+n_{x}+1}$ & 220 & 320 & 230 & 330 & 221 & 321 & 231 & 331 \\
$P_{j+n_{x} n_{y}}$ & 002 & 102 & 012 & 112 & 003 & 103 & 013 & 113 \\
$P_{j+n_{x} n_{y}+1}$ & 202 & 302 & 212 & 312 & 203 & 303 & 213 & 313 \\
$P_{j+n_{x} n_{y}+n_{x}}$ & 022 & 122 & 032 & 132 & 023 & 123 & 033 & 133 \\
$P_{j+n_{x} n_{y}+n_{x}+1}$ & 222 & 322 & 232 & 332 & 223 & 323 & 233 & 333
\end{tabular}

Table 1: Mapping of the coefficients $\left\{b_{i, l m n}\right\}$ (defined in Equation 14) to the field values and derivatives at the grid points contained in the box with lowestindexed point $P_{j}$. Entries in the table are the values of $\operatorname{lm} n$ corresponding to each combination of point and coefficient on the interpolation box. $\mathrm{m}$

\section{mat}

\section{or}

\subsection{Field near the coils}

Some of the interpolation grid points sat very close to line segments of current in the field model, which was problematic since the field diverged there. The field and the field derivatives at these grid points were unreliable, and this spoiled the magnetic field over the eight grid boxes surrounding every such point. When simulating trajectories that pass close to the coils, we observed that particles had abruptly different trajectories if they entered a spoiled grid box. The instrumented region in OLYMPUS extends to approximately $\pm 15^{\circ}$ in azimuth about the $y=0$ plane; aberrant trajectories were observed at an azimuth of only $12^{\circ}$. The problematic points sit near the coils, at $\pm 22.5^{\circ}$.

To safeguard against this problem, we produced a second field grid using a different procedure. We defined a safe region, $\pm 15^{\circ}$ in azimuth from the $y=0$ plane, inside which we trusted the magnetic field model. (Points inside this region were sufficiently far from the coils to avoid problems with the diverging field.) For grid points in this region, the field and its derivatives were calculated as before. For points outside this region, we used an alternative calculation, exploiting the approximate azimuthal symmetry of the magnet. For each outside grid point, we first calculated the field and its derivatives at the point with the same $z$ and same $r$, but on the nearest $\phi= \pm 15^{\circ}$ plane. Derivatives were calculated in cylindrical coordinates, and any with respect to $\phi$ were set to 0 . We then rotated the field and derivative vectors back to the point of interest. 
For example, given a grid point at $\phi=20^{\circ}, r=1 \mathrm{~m}, z=$ $2 \mathrm{~m}$, the field would first be calculated at $\phi=15^{\circ}, r=1 \mathrm{~m}$, $z=2 \mathrm{~m}$. The derivatives $d \vec{B} / d r, d \vec{B} / d z$, and $d^{2} \vec{B} / d r d z$ would be calculated numerically. All other derivatives would be set to 0 . The vectors would then be rotated by $5^{\circ}$ in $\phi$, so as to correspond appropriately for the grid point at $\phi=20^{\circ}, r=1 \mathrm{~m}$, $z=2 \mathrm{~m}$

The grid produced using this procedure was interpolated with the scheme in Section 5. Subsequent tests showed that simulated trajectories were not aberrant, even out to $\phi= \pm 15^{\circ}$, the limits of the OLYMPUS instrumented region. Furthermore these trajectories were essentially the same as those simulated with the field model directly, without interpolation.

\subsection{Beamline region}

The region near the beamline, where the magnetic field was small, was difficult to reproduce accurately using the coil fitting ${ }^{304}$ model for two reasons. The first is that this region is close to ${ }^{305}$ all eight coils. Slight changes in the model's coil placement can ${ }^{306}$ create large gradients in the region. The second is that there ${ }^{307}$ were few measurements made in that region (and none inside ${ }^{308}$ the volume of the target chamber) to constrain the fit. Møller ${ }^{309}$ and Bhabha tracks pass through this region, and an accurate ${ }^{310}$ simulation is necessary for the Møller/Bhabha luminosity mon- ${ }^{311}$ itor (described by Pérez Benito et al. [8]). Since the magnetic ${ }^{312}$ field model failed in this region, a dedicated alternative inter- ${ }^{313}$ polation scheme was developed, for use strictly in this volume. ${ }^{314}$ The region included all points with $|x|<100 \mathrm{~mm},|y|<50 \mathrm{~mm},{ }^{315}$ and $500 \mathrm{~mm}<z<3000 \mathrm{~mm}$, shown in Figure 8.

To provide an accurate field map for the forward ${ }^{317}$ Møller/Bhabha scattering region, interpolation was performed ${ }^{318}$ directly on the measured data points in the region. This data ${ }^{319}$ was approximately located on the $y=0$ plane with small varia- ${ }^{320}$ tions due to the imperfections of the translation table, described ${ }^{321}$ in Section 4. Since the variation of the field in $y$ was very small ${ }^{322}$ in this region $\left(\sim 10^{-4} \mathrm{~T}\right)$, the $y$ variation in the grid was ignored ${ }^{323}$ and a Lagrange polynomial interpolation was used on the re- ${ }^{324}$ maining irregular 2D grid to produce a regular $5 \mathrm{~mm} \times 5 \mathrm{~mm}^{325}$ grid in $x$ and $z$ for each of the three field components in the region [9]. This regular grid was then appended to the field map 326 and used for interpolation of the field in the special region.

During the field measurements, it was not feasible to pass the Hall probe through the walls of the target chamber. Con- ${ }^{328}$ sequently, no measurements were made for points where $|x|<{ }_{330}$ $100 \mathrm{~mm},|y|<50 \mathrm{~mm}$, and $z<500 \mathrm{~mm}$. However, a few field measurements were made in 2011, prior to the installation of the chamber, and these data confirmed that the field inside the chamber is on the order of $10^{-4} \mathrm{~T}$. In this region, we chose to use the standard grid interpolation for $B_{y}$ and $B_{z}$, since the field model reproduces the 2011 measurements well in those directions. For $B_{x}$, a parabolic fit to the 2011 measurements was $^{335}$ chosen for the $x$ dependence, with the assumption that $B_{x}$ is $_{336}$ independent of $y$ and $z$.

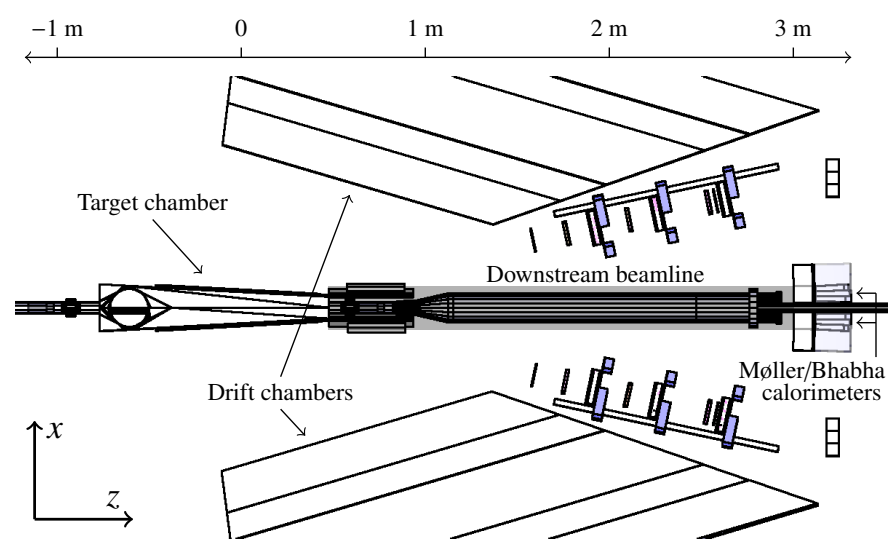

Figure 8: The special beamline field region, shown in the shaded box, contained the entirety of the downstream beam pipe from the target cell to the collimators of the Møller/Bhabha calorimeters.

\section{Conclusions}

We have described the measurement procedure and the data analysis techniques employed to make a comprehensive survey of the OLYMPUS spectrometer's magnetic field. Using an apparatus consisting of a Hall probe, actuated with a system of translation tables, we measured the magnetic field at over 36,000 positions in and around the spectrometer volume. We chose to fit these field data with a numerical field model to calculate the field at arbitrary positions. For analysis applications that required rapid queries of the magnetic field, we precomputed the field and its derivatives on a grid of positions, and developed a scheme to interpolate the field between grid points using tricubic splines. By refactoring the splines, we found that we could reduce the memory needed to store the necessary spline coefficients by a factor of eight. This interpolation scheme worked well for the majority of the spectrometer volume; however, two regions - the region close to the coils, and the region along the beamline-required special adjustments, which we described. By making these adjustments, we succeeded in producing a scheme for determining the magnetic field rapidly and accurately. This is crucial for the OLYMPUS analysis since it allows a high-rate simulation of particle trajectories.

\section{Acknowledgements}

We gratefully acknowledge Philipp Altmann at DESY for his assistance in assembling the measurement apparatus and preparing for the field measurements. We are also very thankful for the expertise of Martin Noak at DESY in surveying and aligning the apparatus.

This work was supported by the Office of Nuclear Physics of the U.S. Department of Energy, grant No. DE-FG0294ER40818.

\section{References}

[1] F. Lekien, J. Marsden, Tricubic interpolation in three dimensions, International Journal for Numerical Methods in Engineering 63 (2005) 455-471. 
[2] R. Milner, D. Hasell, M. Kohl, U. Schneekloth, N. Akopov, et al., The OLYMPUS Experiment, Nuclear Instruments and Methods in Physics Research Section A: Accelerators, Spectrometers, Detectors and Associated Equipment 741 (2014) 1-17.

[3] P. A. Guichon, M. Vanderhaeghen, How to reconcile the Rosenbluth and the polarization transfer method in the measurement of the proton formfactors, Phys.Rev.Lett. 91 (2003) 142303.

[4] P. Blunden, W. Melnitchouk, J. Tjon, Two photon exchange and elastic electron proton scattering, Phys.Rev.Lett. 91 (2003) 142304.

[5] D. Hasell, et al., The BLAST experiment, Nucl. Instrum. Meth. A603 (2009) 247-262.

[6] K. A. Dow, T. Botto, A. Goodhue, D. Hasell, D. Loughnan, K. Murphy, T. P. Smith, V. Ziskin, Magnetic field measurements of the BLAST spectrometer, Nucl. Instrum. Meth. A599 (2009) 146-151.

[7] O. Grimm, N. Morozov, A. Chesnov, Y. Holler, E. Matushevsky, D. Petrov, J. Rossbach, E. Syresin, M. Yurkov, Magnetic measurements with the \{FLASH\} infrared undulator, Nuclear Instruments and Methods in Physics Research Section A: Accelerators, Spectrometers, Detectors and Associated Equipment 615 (2010) $105-113$.

[8] R. Pérez Benito, D. Khaneft, C. O'Connor, L. Capozza, J. Diefenbach, B. Gläser, Y. Ma, F. Maas, D. R. Piñeiro, Design and Performance of a Lead Fluoride Detector as a Luminosity Monitor, arXiv 1602.01702 (2016) physics.ins-det.

[9] P. R. Bevington, D. K. Robinson, Data reduction and error analysis for the physical sciences; 3rd ed., McGraw-Hill, New York, NY, 2003. 